\title{
Generation of Bessel Surface Plasmon Polaritons in a Finite-Thickness Metal Film
}

\author{
S. N. Kurilkina, V. N. Belyi, and N. S. Kazak \\ B.I. Stepanov Institute of Physics of National Academy of Sciences of Belarus, 68 Nezavisimosti Avenue, 220072 Minsk, Belarus
}

Correspondence should be addressed to S. N. Kurilkina; s.kurilkina@ifanbel.bas-net.by

Received 9 January 2013; Revised 11 March 2013; Accepted 23 March 2013

Academic Editor: Yu S. Kivshar

Copyright (c) 2013 S. N. Kurilkina et al. This is an open access article distributed under the Creative Commons Attribution License, which permits unrestricted use, distribution, and reproduction in any medium, provided the original work is properly cited.

A theory of generation of low- and high-index Bessel surface plasmon polaritons and their superposition in a metal film of a finite thickness is developed. Correct analytical expressions are obtained for the field of two families of Bessel surface plasmon polariton modes formed inside and outside the metal layer. The intensity distribution near the boundary of the layer has been calculated and analyzed. A scheme for the experimental realization of a superposition of Bessel surface plasmon polaritons is suggested. Our study demonstrates that it is feasible to use the superposition of Bessel surface plasmon polaritons as a virtual tip for near-field optical microscopy with a nanoscale resolution.

\section{Introduction}

Surface plasmon polaritons (SPPs) are surface electromagnetic waves related to collective electron oscillations near the metal surface excited by light [1-3]. These fields arise under the resonance condition, and due to such nature of excitation, they are attractive in the context of enhancing the resolution of imaging systems substantially by increasing the amplitude of evanescent waves [4-6].

In 1987, Durnin et al. suggested a new type of waves, namely, Bessel light beams (BLBs), that kept the transverse spot size unchanged much longer than the Rayleigh range $[7,8]$. Such a localized radiation mode was called the nondiffracting beam (or the diffraction-free beam). The transverse profile of the amplitude of this beam is described by a Bessel function of the first kind. In the domain of spatial frequencies, BLB is represented as a superposition of plane waves which are wrapped around a conical surface. Within the last decades, an intensive study of the scalar and vector BLBs has been made theoretically and experimentally (see, e.g., [9-18]). Bessel light beams are used in numerous applications, such as the optical manipulation of microsized particles [19], the fabrication of long polymer fiber induced by the photopolymerization [20] and microchanneling by structural modification in glass materials [21] the enhancement of energy gain in inverse-free electron lasers and inverse Cherenkov accelerators [22].

The authors of [23] pioneered in obtaining solutions of Maxwell's equations which correspond to evanescent BLBs formed in the condition of the internal total reflection in an optically less dense dielectric medium. A more detailed theory of evanescent Bessel beams is presented in [2429]. These beams exponentially decay while moving off the surface but retain their original transversal shape. In those investigations, of particular interest was the structure of the central lobe of evanescent BLBs. It is established that its diameter can be reduced to a nanosize value. This makes it possible to use evanescent Bessel beams in optical microscopy [30].

But the evanescent BLBs investigated before [24-29] possess an essential disadvantage, namely, they are weak, which causes the necessity of application of strong laser fields for their generation. One of the ways of taking Bessel light field advantages for microscopy is the formation of Bessel surface plasmon polaritons (BSPPs). Kano et al. [31] reported the first experimental result concerning an efficient excitation of surface plasmon polaritons by using the zerothorder BLBs. More recently, radially polarized BLBs have been 
demonstrated to provide the TM polarization required for an effective coupling to the SPPs, which can be used as a virtual probe for the two-photon fluorescence microscopy [32]. In paper [33], the authors have demonstrated the existence of SPP in a multilayered metal-dielectric structure that evolves without distortion within an energy attenuation length and whose in-plane profile traces a Bessel function along the transverse direction relative to the beam axis.

However, despite of intensive theoretical and experimental investigations up to now there is no detailed analysis of the problem of generation of different types of Bessel surface plasmon polaritons in the structure including a metallic absorbing layer separating a substrate and targeted (dielectric) medium. Also of great interest is the study of the intensity distribution in the structure containing a thin metal film. An exact solution of these problems is presented in this paper.

The study of the superposition of two vortex Bessel surface plasmon polaritons with opposite topological charges $\left(J_{m}\right.$ and $\left.J_{-m}\right)$ is undoubtedly beneficial in practical terms. Such superposed beams are promising for creating near the interface an array of diffraction-free nanoscale light needles. At the same time, we attach importance to the development of a scheme for experimental realization of a superposition of Bessel surface plasmon polaritons. And the theory developed ought to be best suited to experiment. This practical motivation necessitates a more detailed description of the field which is a superposition of Bessel surface plasmon polaritons.

The paper is structured as follows. In Section 2, the description of features of generation of different types of Bessel surface plasmon polaritons in layered metal-dielectric structure is presented. The intensity distribution in the structure is analyzed in details analytically and numerically in Section 3. The possibility of the use of a superposition of Bessel surface plasmon polaritons is considered in Section 4. A conclusion is given in Section 5.

\section{Description of Bessel Surface Plasmon Polaritons Generation in a Metal-Dielectric Layered Structure}

\subsection{Formation of Bessel Surface Plasmon Polaritons in a Finite-} Thickness Metal Film. Let us consider a vector TM-polarized high-order $(m \neq 0)$ Bessel light beam $J_{m}$ (BLB) incident onto the boundary of two semi-infinite media. We use the cylindrical coordinate system with the unit vectors $\vec{e}_{\rho}, \vec{e}_{\varphi}$, and $\vec{e}_{z} \vec{e}_{z}$ is collinear to the propagation direction). It is known (see, e.g., $[10,17,18]$ ) that the electric $\vec{E}(R)$ and magnetic $\vec{H}(R)$ vectors of BLB, propagating normally (in $z$ direction) in a medium with the refractive index $n_{i}$, can be represented as

$$
\begin{aligned}
\vec{E}(R) & =A \vec{E}^{\mathrm{TM}}(\rho) \exp i\left(k_{z i} z+m \varphi\right), \\
\vec{H}(R) & =A \vec{H}^{\mathrm{TM}}(\rho) \exp i\left(k_{z i} z+m \varphi\right),
\end{aligned}
$$

where $R=(\rho, \varphi, z)$ are the cylindrical coordinates, $m$ is an integer, $k_{z i}$ is the $z$-component of the wave vector, $A$ is a complex constant, the common phase multiplier $\exp [i(q x-$ $\omega t)$ ] is omitted, and $q=\sqrt{k_{0}^{2} n_{i}{ }^{2}-k_{z i}^{2}}$ is the transversal component of the wave vector.

Using the solution of Maxwell's equations for the components of the vector amplitudes $\vec{E}^{\mathrm{TM}}(\rho), \vec{H}^{\mathrm{TM}}(\rho)$, the problem of transmission and reflection of vector Bessel beams at the boundary of two dielectrics has been solved (see $[17,18]$ ). Following $[17,18]$, in the case of TM-polarized BLB with an amplitude $A_{\text {inc }}$ incident on the interface between two media characterized by refractive indices $n_{i}$ and $n_{j}$, the electric $\vec{E}_{t}(R)$ and magnetic $\vec{H}_{t}(R)$ vectors of the refracted Bessel beam (symbol " $t$ " denotes refracted BLB) can be represented in the form

$$
\begin{gathered}
\vec{E}_{t}(R)=\vec{E}_{t}^{\mathrm{tr}}(R)+\vec{E}_{t}^{l}(R), \\
\vec{E}_{t}^{l}(R)=A_{\text {inc }} t_{i j}^{\mathrm{TM}} \exp i\left[m \varphi+k_{z j} z\right] \sin \gamma_{j} J_{m}(q \rho) \vec{e}_{z}, \\
\vec{E}_{t}^{\mathrm{tr}}(R)=\frac{i A_{\mathrm{inc}}}{\sqrt{2}} \exp i\left[(m-1) \varphi+k_{z j} z\right] t_{i j}^{\mathrm{TM}} \\
\times \cos \gamma_{j}\left[J_{m-1}(q \rho) \vec{e}_{+}-J_{m+1}(q \rho) \exp (2 i \varphi) \vec{e}_{-}\right] \\
\vec{H}_{t}(R)=\frac{n_{j} A_{\text {inc }}}{\sqrt{2}} \exp i\left[(m-1) \varphi+k_{z j} z\right] t_{i j}^{\mathrm{TM}} \\
\times\left[J_{m-1}(q \rho) \vec{e}_{+}+J_{m+1}(q \rho) \exp (2 i \varphi) \vec{e}_{-}\right] .
\end{gathered}
$$

Here $\cos \gamma_{j}=k_{z j} / k_{0} n_{j}, k_{0}=\omega / c, \omega$ is the frequency of the Bessel beam, $c$ is the light velocity in vacuum, $J_{m}(q \rho)$ is the $m$-order Bessel function; symbols "tr," "l" denote the transversal and longitudinal component of the electric (magnetic) vector, respectively; $t_{i j}^{\mathrm{TM}}=A_{t}^{\mathrm{TM}} / A_{\mathrm{inc}}$ is the amplitude transmission coefficient; $A_{t}^{\mathrm{TM}}$ is the amplitude factor for TM BLB; $\vec{e}_{ \pm}=\left(\vec{e}_{1} \pm i \vec{e}_{2}\right) / \sqrt{2}$ are the unit circular vectors which are orthogonal to the $\vec{e}_{z}$ vector.

In a similar manner, for the reflected field (it is marked with the symbol " $r$ "), we obtain

$$
\begin{gathered}
\vec{E}_{r}(R)=\vec{E}_{r}^{\mathrm{tr}}(R)+\vec{E}_{r}^{l}(R), \\
\vec{E}_{r}^{l}(R)=A_{\mathrm{inc}} r_{i j}^{\mathrm{TM}} \exp i\left[m \varphi-k_{z i} z\right] \sin \gamma_{i} J_{m}(q \rho) \vec{e}_{z}, \\
\vec{E}_{r}^{\mathrm{tr}}(R)=-\frac{i A_{\mathrm{inc}}}{\sqrt{2}} \exp i\left[(m-1) \varphi-k_{z i} z\right] r_{i j}^{\mathrm{TM}} \\
\times \cos \gamma_{i}\left[J_{m-1}(q \rho) \vec{e}_{+}-J_{m+1}(q \rho) \exp (2 i \varphi) \vec{e}_{-}\right],
\end{gathered}
$$

$$
\begin{aligned}
\vec{H}_{r}(R)= & \frac{n_{i} A_{\text {inc }}}{\sqrt{2}} \exp i\left[(m-1) \varphi-k_{z i} z\right] r_{i j}^{\mathrm{TM}} \\
& \times\left[J_{m-1}(q \rho) \vec{e}_{+}+J_{m+1}(q \rho) \exp (2 i \varphi) \vec{e}_{-}\right] .
\end{aligned}
$$




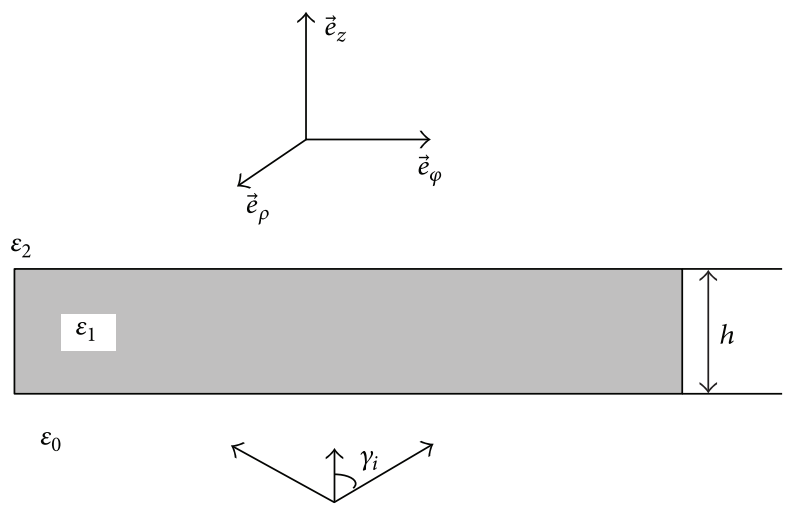

FIGURE 1: Schematic representation of the structure including a metallic layer $\left(\varepsilon_{1}\right)$, a substrate $\left(\varepsilon_{0}\right)$, and a targeted $\left(\varepsilon_{2}\right)$ medium.

Here $r_{i j}^{\mathrm{TM}}=A_{r}^{\mathrm{TM}} / A_{\text {inc }}$ is the amplitude reflection coefficient; $A_{r}^{\mathrm{TM}}$ is the amplitude factor for TM BLB. The amplitude refraction and reflection coefficients are

$$
\begin{aligned}
t_{i j}^{\mathrm{TM}} & =\frac{2 n_{i} \cos \gamma_{i}}{n_{i} \cos \gamma_{j}+n_{j} \cos \gamma_{i}}, \\
r_{i j}^{\mathrm{TM}} & =\frac{n_{j} \cos \gamma_{i}-n_{i} \cos \gamma_{j}}{n_{j} \cos \gamma_{i}+n_{i} \cos \gamma_{j}} .
\end{aligned}
$$

Equations (6) can be used for the description of the transformation of BLB at the boundary between dielectric with the real refractive index $n_{i}$ and metal having the complex refractive index $n_{j}=n_{j}^{\prime}+i n_{j}^{\prime \prime}$, where $n_{j}^{\prime}=\operatorname{Re}\left(n_{j}\right), n_{j}^{\prime \prime}=$ $\operatorname{Im}\left(n_{j}\right)$.

Using (2), (3), and (6), let us consider now the formation of Bessel surface plasmon polaritons in a layered structure containing a metal film with the dielectric constant $\varepsilon_{1}=n_{1}^{2}$ and thickness $h$ between two semi-infinite dielectric media with dielectric constants $\varepsilon_{0}=n_{0}^{2}, \varepsilon_{2}=n_{2}^{2}$ (Figure 1). The origin of the coordinated system $z=0$ is assumed to be chosen at the interface between the dielectric substrate and the layer $\varepsilon_{1}$ (Figure 1 ).

In view of (2), (4), the electric vector of the field inside the metal layer and outside it is expressed as

$$
\begin{gathered}
\vec{E}_{0}(R)=\vec{E}_{0}^{\mathrm{tr}}(R)+\vec{E}_{0}^{l}(R), \\
\vec{E}_{0}^{l}(R)=A_{\mathrm{inc}} \frac{q}{k_{0} n_{0}} \exp i\left[m \varphi-k_{z 0} z\right] J_{m}(q \rho) \vec{e}_{z},
\end{gathered}
$$

$\vec{E}_{0}^{\operatorname{tr}}(R)$

$=-\frac{i A_{\text {inc }}}{\sqrt{2}} \exp i\left[(m-1) \varphi-k_{z 0} z\right]$

$\times \sqrt{1-\left(\frac{q}{k_{0} n_{0}}\right)^{2}}\left[J_{m-1}(q \rho) \vec{e}_{+}-J_{m+1}(q \rho) \exp (2 i \varphi) \vec{e}_{-}\right]$,

$$
\vec{E}_{2}(R)=\vec{E}_{2}^{\mathrm{tr}}(R)+\vec{E}_{2}^{l}(R),
$$

$\vec{E}_{2}^{l}(R)=A_{\mathrm{inc}} \frac{q}{k_{0} n_{2}} t \exp i\left[m \varphi+k_{z 2}(z-h)\right] J_{m}(q \rho) \vec{e}_{z}$,
$\vec{E}_{2}^{\mathrm{tr}}(R)$

$=\frac{i A_{\mathrm{inc}}}{\sqrt{2}} \exp i\left[(m-1) \varphi+k_{z 2}(z-h)\right] t$

$\times \sqrt{1-\left(\frac{q}{k_{0} n_{2}}\right)^{2}}\left[J_{m-1}(q \rho) \vec{e}_{+}-J_{m+1}(q \rho) \exp (2 i \varphi) \vec{e}_{-}\right]$,

$\vec{E}_{1}(R)=\vec{E}_{1}^{\mathrm{tr}}(R)+\vec{E}_{1}^{l}(R)$,

$\vec{E}_{1}^{l}(R)=\left(\vec{E}_{1}^{l}\right)^{f}+\left(\vec{E}_{1}^{l}\right)^{b}$,

$\vec{E}_{1}^{\mathrm{tr}}(R)=\left(\vec{E}_{1}^{\mathrm{tr}}\right)^{f}+\left(\vec{E}_{1}^{\mathrm{tr}}\right)^{b}$,

$\left(\vec{E}_{1}^{l}\right)^{f, b}=A_{\text {inc }} \frac{q}{k_{0} n_{1}} s^{f, b} \exp i\left[m \varphi \pm k_{z 1} z\right] J_{m}(q \rho) \vec{e}_{z}$,

$$
\begin{aligned}
& \left(\vec{E}_{1}^{\mathrm{tr}}(R)\right)^{f, b} \\
& = \pm \frac{i A_{\text {inc }}}{\sqrt{2}} s^{f, b} \exp i\left[(m-1) \varphi \pm k_{z 1} z\right] \\
& \times \sqrt{1-\left(\frac{q}{k_{0} n_{1}}\right)^{2}}\left[J_{m-1}(q \rho) \vec{e}_{+}-J_{m+1}(q \rho) \exp (2 i \varphi) \vec{e}_{-}\right]
\end{aligned}
$$

Here, symbols " 0 ", "1", and " 2 " denote the substrate, metal layer, and targeted medium, respectively. Similarly, for the magnetic vector we have

$$
\begin{aligned}
\vec{H}_{0}(R)= & \frac{n_{0} A_{\text {inc }}}{\sqrt{2}} \exp i\left[(m-1) \varphi-k_{z 0} z\right] \\
& \times\left[J_{m-1}(q \rho) \vec{e}_{+}+J_{m+1}(q \rho) \exp (2 i \varphi) \vec{e}_{-}\right],
\end{aligned}
$$




$$
\begin{aligned}
\vec{H}_{2}(R)= & \frac{n_{2} A_{\text {inc }}}{\sqrt{2}} \exp i\left[(m-1) \varphi+k_{z 2}(z-h)\right] \\
& \times t\left[J_{m-1}(q \rho) \vec{e}_{+}+J_{m+1}(q \rho) \exp (2 i \varphi) \vec{e}_{-}\right] \\
& \vec{H}_{1}(R)=\left(\vec{H}_{1}(R)\right)^{f}+\left(\vec{H}_{1}(R)\right)^{b} \\
\left(\vec{H}_{1}(R)\right)^{f, b}= & \frac{n_{1} A_{\text {inc }}}{\sqrt{2}} s^{f, b} \exp i\left[(m-1) \varphi \pm k_{z 1} z\right] \\
& \times\left[J_{m-1}(q \rho) \vec{e}_{+}+J_{m+1}(q \rho) \exp (2 i \varphi) \vec{e}_{-}\right]
\end{aligned}
$$

Here, $t=A_{2} / A_{\text {inc }}, s^{f}=A_{f} / A_{\text {inc }}, s^{b}=A_{b} / A_{\text {inc }}$ are the amplitude coefficients for the forward and backward Bessel fields inside the metal film, respectively; $A_{f, b}$ are the amplitude factors inside the metal layer.

Using the boundary conditions of continuity of the tangential components of the electric and magnetic fields, which have to be satisfied at the planes $z=0$ and $z=$ $h$, as well as (7a), (7b), (8a), (8b), and (6), we obtain the system of equations for the coefficients $t, s^{f, b}$. As a result of its solution, one can find the dispersion equation determining the existence of Bessel surface plasmon polaritons in the structure shown in Figure 1:

$$
1+r_{01}^{\mathrm{TM}} r_{12}^{\mathrm{TM}} \exp 2 i\left(k_{z 1} h\right)=0
$$

As known (see [34]), the amplitude reflection coefficient of the layer is

$$
r=\frac{r_{01}^{\mathrm{TM}}+r_{12}^{\mathrm{TM}} \exp 2 i\left(k_{z 1} h\right)}{1+r_{01}^{\mathrm{TM}} r_{12}^{\mathrm{TM}} \exp 2 i\left(k_{z 1} h\right)} .
$$

Then, (9) determines the poles of the reflection coefficient $r$ of the metal layer. Taking into account (6), this expression can be transformed to the form

$$
F=-i \operatorname{tg}\left(k_{z 1} h\right)\left(1+\frac{k_{z 1}^{2} \varepsilon_{0} \varepsilon_{2}}{k_{z 0} k_{z 2} \varepsilon_{1}^{2}}\right)+\frac{k_{z 1} \varepsilon_{0}}{k_{z 0} \varepsilon_{1}}+\frac{k_{z 1} \varepsilon_{2}}{k_{z 2} \varepsilon_{1}}=0 .
$$

Here, $k_{z i}$ is the $z$-component of the wave vector, and the index $i$ refers to the medium number. As seen from (11), unlike plane waves, the dispersion equation relates the frequency with the transversal component $q=\sqrt{k_{0}^{2} n_{j}^{2}-k_{z j}^{2}}$ of a Bessel surface plasmon polariton. Due to complexity of the metal refractive index, $q$ is a complex quantity. Here, Re $q$ determines the radius of rings of the excited Bessel surface plasmon polariton.
The boundary conditions allow one to determine the quantities $t, s^{f}$, and $s^{b}$ entering into (7a), (7b), (8a), and (8b):

$$
\begin{gathered}
t=\frac{t_{01}^{\mathrm{TM}} t_{12}^{\mathrm{TM}} \exp \left(i k_{z 1} h\right)}{1+r_{01}^{\mathrm{TM}} r_{12}^{\mathrm{TM}} \exp \left(2 i k_{z 1} h\right)}, \\
s^{f}=\frac{t_{01}^{\mathrm{TM}}}{1+r_{01}^{\mathrm{TM}} r_{12}^{\mathrm{TM}} \exp \left(2 i k_{z 1} h\right)}, \\
s^{b}=\frac{t_{01}^{\mathrm{TM}} r_{12}^{\mathrm{TM}} \exp \left(2 i k_{z 1} h\right)}{1+r_{01}^{\mathrm{TM}} r_{12}^{\mathrm{TM}} \exp \left(2 i k_{z 1} h\right)} .
\end{gathered}
$$

Here, the coefficients $t_{i j}^{\mathrm{TM}}, r_{i j}^{\mathrm{TM}}$ are determined by (6).

Then, (7a), (7b), (8a), (8b), and (12) at $q$, which meets condition (11), describe Bessel surface plasmon polaritons formed in the structure "dielectric substrate metal layer dielectric." The field of a superposition of Bessel surface plasmon polaritons can be defined as a sum of the beams with the electric (magnetic) vector in the form $(7 \mathrm{a}),(7 \mathrm{~b})((8 \mathrm{a})$, and (8b)).

It would be interesting to give a physical interpretation of the Bessel SPPs being localized and to compare them with traditional surface plasmon polaritons. It is well-known that the traditional SPPs are associated with the propagating wave process on metal/dielectric surface. In contrast to this, according to (7a), (7b), (8a), and (8b), the Bessel SPP is excited by an incident BLB, which can be considered as a superposition of $p$-polarized plane monochromatic waves having frequency $\omega$ and wave vectors lying on the surface of a cone.

The SPP propagating in the metal film is excited by every $p$-polarized plane-wave component of the incident BLB in conditions of the plasmon resonance. Here, the phase of every SPP is determined by the phase of the plane-wave component of the incident BLB. Thus, in the metal film, an array of propagating SPPs arises, wave vectors of which are being oriented in the direction of the center determining the intersection of the incident BLB axis with the surface of the metal film. As a result, there occurs the generation of pairs of two counter-propagating SPP waves with the wave vectors $\pm \vec{q}$. The generated SPPs will propagate in all the possible radial directions to form a localized SPP field. This results in a complex high-symmetric interference light structure emerging in sections parallel to the metal-dielectric surface.

For the case where the longitudinal components of the electric field $\vec{E}_{1}^{l}$ for every radially propagating SPP has the same phase, in the center of such a standing light structure a maximum appears, that is, the shape of the $\vec{E}_{1}^{l}$ component of localized Bessel SPP is described by zero-order Bessel function $J_{0}(q \rho)$. In a special case, each pair of counter propagating SPPs is in counter-phase and the local plasmonic field appears with a minimum in the center, that is, the socalled vortex localized Bessel SPP is formed. In this case, the electric field $\vec{E}_{1}^{l}$ of vortex Bessel SPP is described by $J_{m}(q \rho)$ Bessel function (see (7b)).

The problem should be studied now of attenuation of the Bessel SPP inside the metal layer. With this aim, the limited narrow Bessel light beam of radius $r_{0}$ in the 


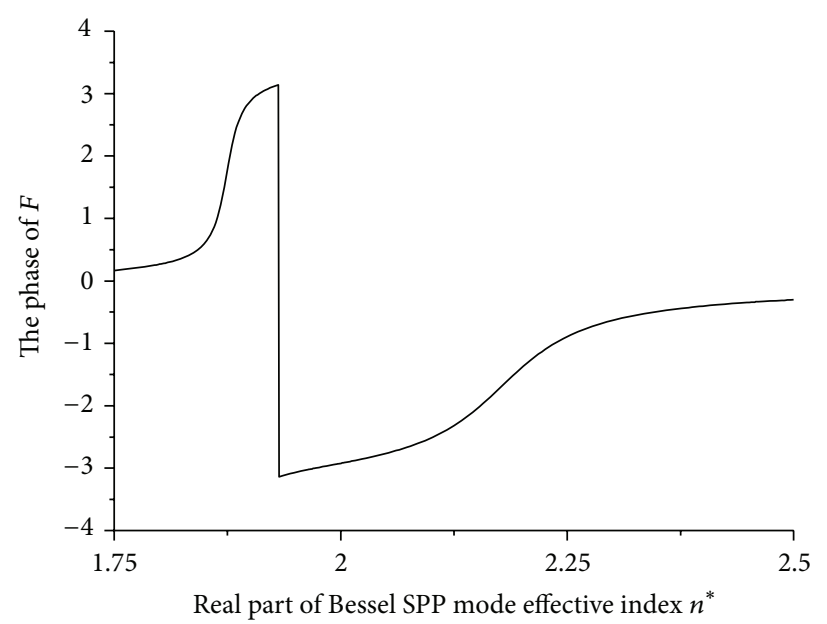

(a)

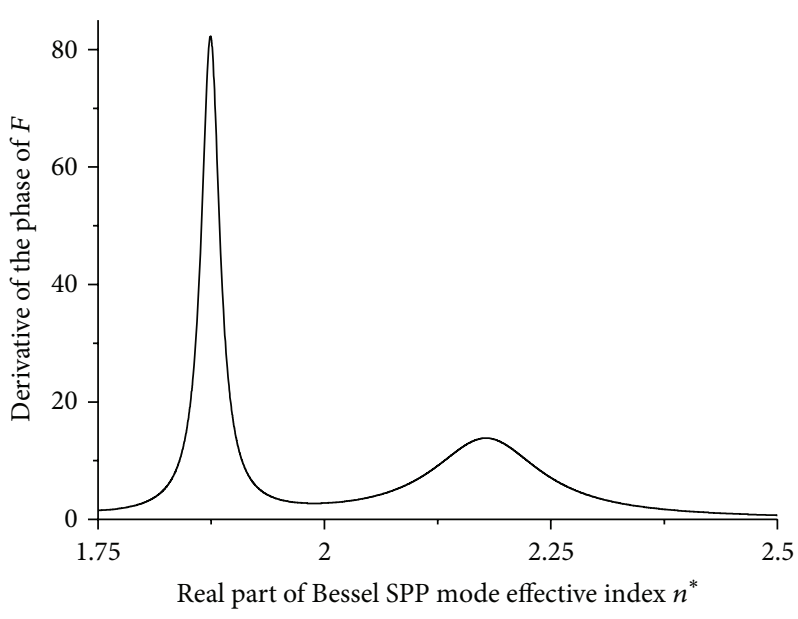

(b)

Figure 2: The phase of $F$ function (a) and the derivative of the phase of $F$ function (b) versus the real part of the Bessel surface plasmon polariton mode effective index.

transversal section should be considered. Such an apertured beam excites Bessel SSP in a cylindrical region limited by $r_{0}$. As follows from (7a), (7b), the transversal distribution of the longitudinal component of the electric field of Bessel SPP is determined by $J_{m}(q \rho)$ Bessel function which can be represented as a sum of the cylindrical Hankel functions of the first $H_{m}^{(1)}(q \rho)$ (outgoing) and second $H_{m}^{(2)}(q \rho)$ (incoming) kinds [35]:

$$
J_{m}(q \rho)=\frac{\left(H_{m}^{(1)}(q \rho)+H_{m}^{(2)}(q \rho)\right)}{2} .
$$

According to (13), in the region $\rho<r_{0}$, the Bessel SPPs are formed by converging and diverging conical beams described by appropriate Hankel functions. Outside the area $\rho>r_{0}$ there exists only a diverging conical beam, and that is why in the decomposition (13) it is supposed that $H_{m}^{(2)}=0$. Using the asymptotic approximation of Hankel function [36], we obtain that the intensity of the longitudinal component of Bessel SPP electric vector $\left|\vec{E}^{l}\right|^{2} \sim\left|J_{m}^{2}(q \rho)\right|^{2}$ is determined by the expression

$$
\left|\vec{E}^{l}\right|^{2} \sim \frac{1}{|q|^{2} \rho^{2}} \exp [-2(\operatorname{Im} q) \rho] .
$$

Thus, from (14), it follows that beyond the boundary of the exciting source the Bessel SPP decays exponentially in the radial direction. The $1 / e$ energy-attenuation radius $R_{\mathrm{BSSP}}$ is determined by the imaginary part of the SPP wave number and given by the expression

$$
R_{\mathrm{BSSP}}=\frac{1}{2 \operatorname{Im} q}
$$

Thus, the energy-attenuation radius of Bessel SPP, generated by limited narrow Bessel beam, coincides with the decay length $L_{\mathrm{SP}}$ of propagating surface plasmon. From the physical point of view, it is explained by the fact that the Bessel SPP (outside the area of excitation) can be represented by the superposition of radially propagating plane wave SPPs.

2.2. Formation of Bessel Surface Plasmon Polaritons in a Symmetric Metal-Dielectric Layered Structure. Finding the roots of the complex equation (11) is numerically challenging. The reflection pole method (RPM) provides the phase of $F$ as a function of the real part of the Bessel surface plasmon polariton mode effective index $n^{*}=q / k_{0}$. The Bessel surface plasmon polaritons correspond to the rapid changes of the phase of $F$ function. According to the Bode plot theory [37], a peak of the derivative of the phase of $F$ function corresponds to the real part of the Bessel surface plasmon polariton mode effective index, and the full width at the half maximum of the phase derivative curve is the imaginary part of the Bessel surface plasmon polariton mode effective index.

Using the reflection pole method, we searched the Bessel surface plasmon polaritons for a gold $(\mathrm{Au})$ metal film $\left(\varepsilon_{1}=\right.$ $-11.679+1.181 i$ at the 0.633 micron wavelength [38]) of $50 \mathrm{~nm}$ thickness embedded in SF10 glass $\left(\varepsilon_{0}=\varepsilon_{2}=(1.723)^{2}\right)$. Figures 2(a) and 2(b) are the plots of the phase of $F$ function and its derivative versus the real part of the Bessel surface plasmon polariton mode effective index. From Figures 2(a) and 2(b), we see two rapid changes of the phase of $F$ which correspond to two Bessel surface plasmon polariton modes. The complex Bessel surface plasmon polariton mode effective indices we found are $n_{1}^{*}=1.874+0.012 i$ and $n_{2}^{*}=2.179+$ $0.073 i$.

The first (so-called low-index Bessel surface plasmon polariton (LIBSPP)) and the second (high-index Bessel surface plasmon polariton (HIBSPP)) BSPPs are characterized by the first ring radius $R_{1} \approx 2.4 /\left(k_{0} \operatorname{Re} n^{*}\right)$ equal to $129 \mathrm{~nm}$ and $110 \mathrm{~nm}$, respectively.

It is interesting to consider the symmetry of the longitudinal $\left(\vec{E}_{1}^{l}=E_{1}^{l} \vec{e}_{z}\right)$ and transversal, for example, radial $\left(E_{1}^{\rho}\right)$ 


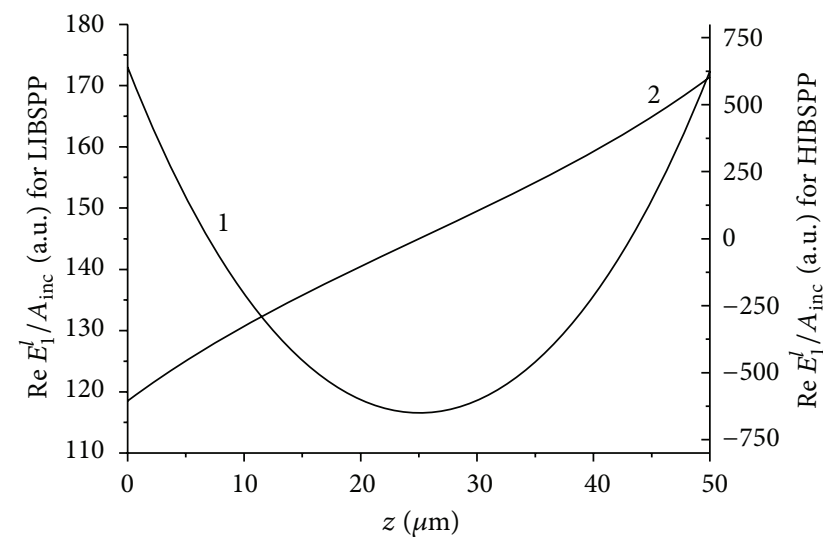

(a)

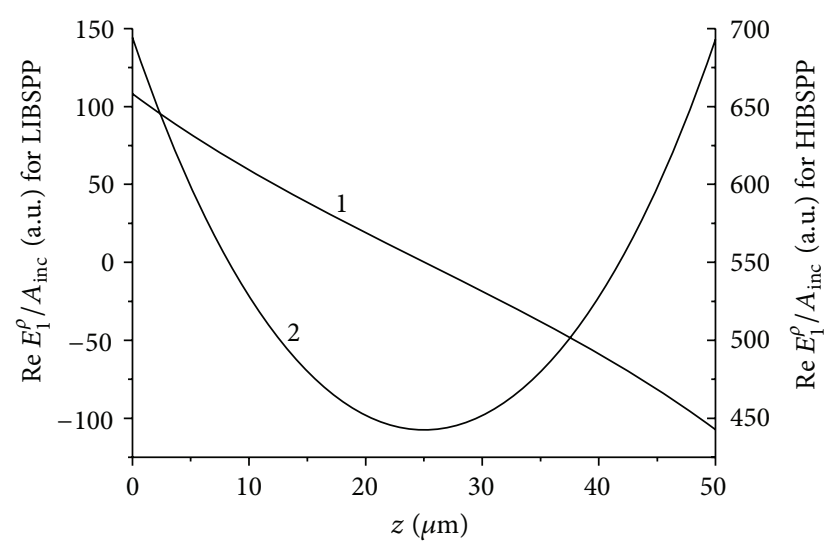

(b)

FIGURE 3: The normalized real part of the longitudinal $E_{1}^{l}$ (a) and radial $E_{1}^{\rho}$ (b) components of the electric vector for low-index (1) and highindex (2) TM-polarized zero-order Bessel surface plasmon polaritons versus the distance inside the gold film of $50 \mathrm{~nm}$ thickness embedded in SF10 glass. The distance from the Bessel surface plasmon polariton axis is $\rho=0.05 \mu \mathrm{m}$, and the wavelength is $\lambda=0.633 \mu \mathrm{m}$.

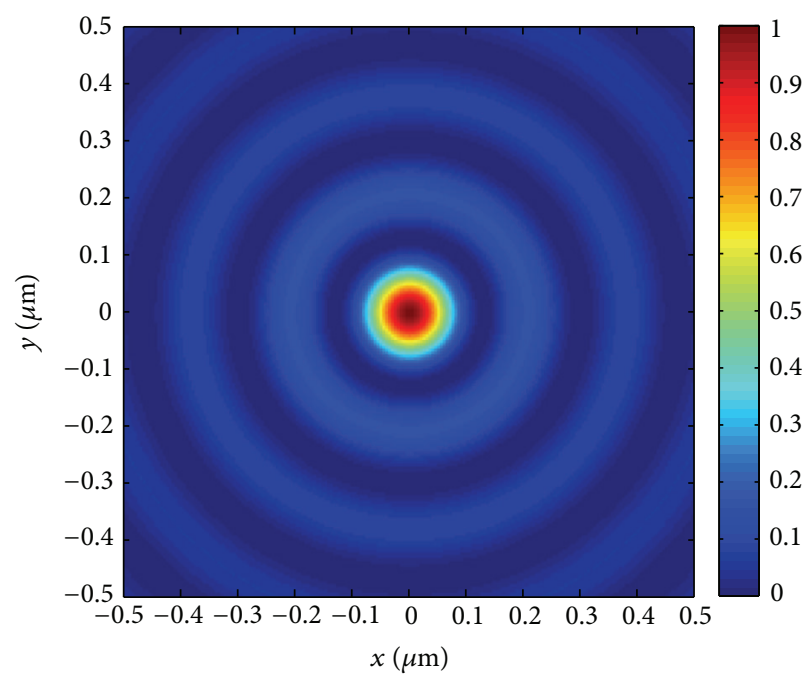

(a)

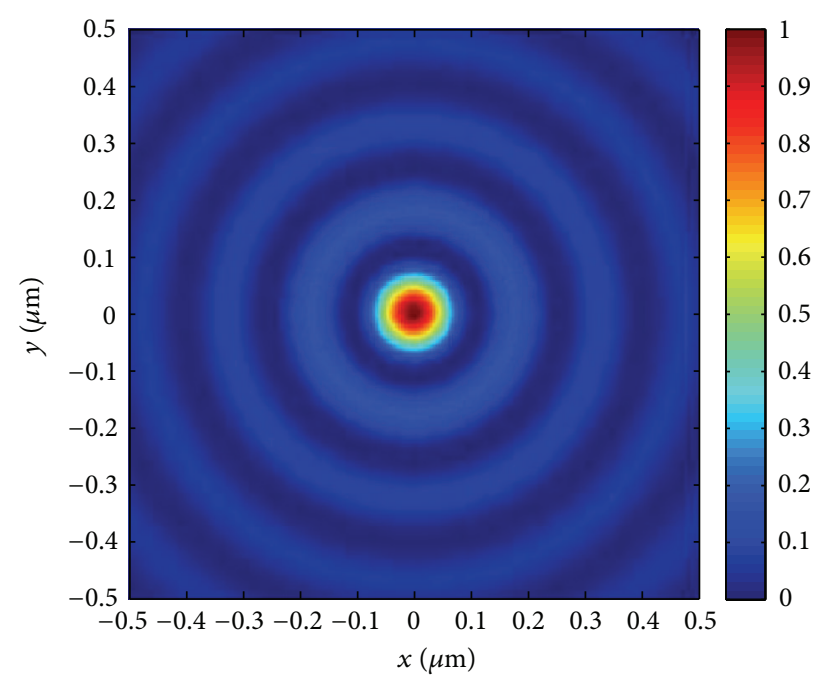

(b)

FIGURE 4: 2D pattern of normalized intensity distribution $\left|E_{2}^{l}\right|^{2} /\left|E_{2}^{l}\right|_{\max }^{2}$ for low- (a) and high-index (b) zero-order Bessel surface plasmon polaritons outside the gold layer of $50 \mathrm{~nm}$ thickness embedded in SF10 glass. BSPPs are generated by a purely radially polarized Bessel beam having a wavelength of $633 \mathrm{~nm}$. The distance from the entrance surface of the metal layer is $z=\lambda / 3$.

components of the BSPP electric vector inside the metal layer. Note that the equation for $E_{1}^{\rho}$ can be obtained from (7b). As calculation shows, the symmetry of real and imaginary parts of longitudinal (as well as transversal) components of electric vector of Bessel SPP inside the metal layer is the same. Figure 3 illustrates the dependence of the function Re $E_{1}^{l}$ normalized to the amplitude factor $A_{\text {inc }}$ (at the center $\rho=0$ of Bessel surface plasmon polariton) on the distance $z$ in a $50 \mathrm{~nm}$ thickness gold film surrounded by dielectric media for low- and high-index zero-order BSPPs. It is seen that for the low-index Bessel SPP with the topological charge $m=0$, the distribution of $E_{1}^{l}\left(E_{1}^{\rho}\right)$ turns out to be symmetrical (antisymmetrical) (Figure 3 ) with respect to the middle plane of the metal film. For the high-index Bessel SPP, it is antisymmetrical (symmetrical).

Figure 4 demonstrates a 2D-pattern of the normalized intensity distribution $\left|E_{2}^{l}\right|^{2} /\left|E_{2}^{l}\right|_{\max }^{2}$ for low- and high-index Bessel surface plasmon polaritons formed outside the gold layer of $50 \mathrm{~nm}$ thickness embedded in SF10 glass. This is the most interesting case where BSPPs are generated by a purely radially polarized Bessel beam. The results of calculations presented in Figure 4 prove that the widths of the central maximum in the transverse distribution, which can be estimated approximately by the ratio $D_{1}=2 R_{1}=$ $4.8 /\left(k_{0} \operatorname{Re}\left(n^{*}\right)\right)$, for LIBSPP as well as for HIBSPP, turn out to be less than the wavelength. It should be noted that as 


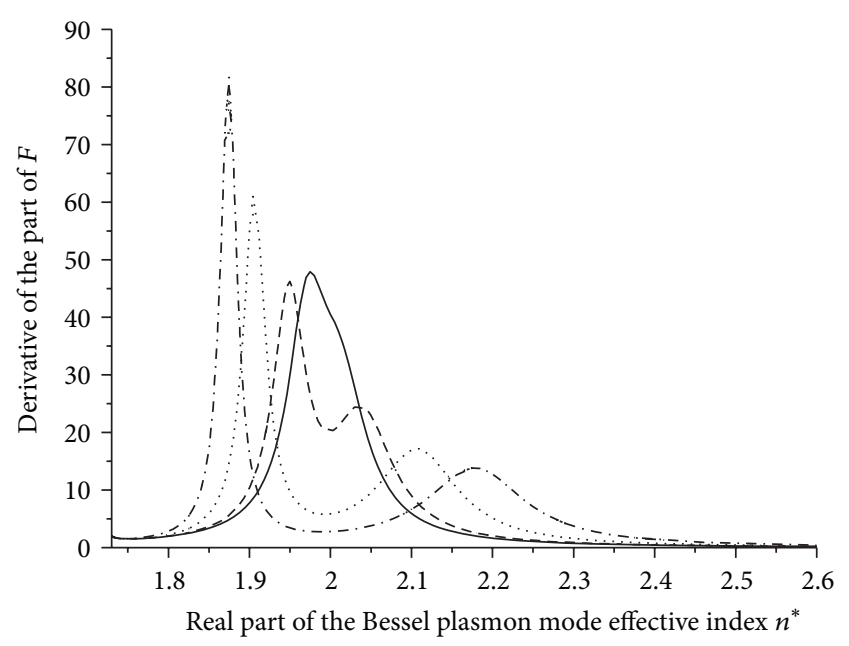

FIgURE 5: The derivative of the phase of $F$ function versus the real part of Bessel surface plasmon polariton mode effective index for gold film of $100 \mathrm{~nm}(-), 80 \mathrm{~nm}(---), 60 \mathrm{~nm}(\cdots)$, and $50 \mathrm{~nm}(\cdot-\cdot-\cdot)$ embedded in SF10 glass.

follows from (7a) while increasing the distance from the surface of the metal layer, the central peak of BSPP, generated in the structure, decays as $\exp \left(-\operatorname{Im} k_{z i} z\right)(i=1,2)$, but its transversal size $D_{1}$ does not change practically.

Let us investigate now how the conditions of excitation of Bessel surface plasmon polaritons depend on the thickness of the metal layer. Figure 5 shows the dependence of the derivative of the phase of $F$ function versus the real part of the Bessel surface plasmon polariton mode effective index at various thicknesses of the gold film. It is seen that at an increase in the metal layer thickness two maxima of the curve merge into one, which testifies the degeneration of Bessel surface plasmon polariton modes. Here, the Bessel surface plasmon polariton mode effective index approaches the $n_{\text {free }}^{*}$ for the case of BSPP excitation at the boundary of two semiinfinite media-dielectric and metal [38]:

$$
n_{\text {free }}^{*}=\sqrt{\varepsilon_{2} \frac{\varepsilon_{1}^{\prime}\left(\varepsilon_{1}^{\prime}+\varepsilon_{2}\right)+\varepsilon_{1}^{\prime \prime 2}+i\left[\varepsilon_{1}^{\prime \prime}\left(\varepsilon_{1}^{\prime}+\varepsilon_{2}\right)-\varepsilon_{1}^{\prime} \varepsilon_{1}^{\prime \prime}\right]}{\left(\varepsilon_{1}^{\prime}+\varepsilon_{2}\right)^{2}+\varepsilon_{1}^{\prime \prime 2}}},
$$

where $\varepsilon_{1}^{\prime}=n_{1}^{\prime 2}-n_{1}^{\prime \prime 2}, \varepsilon_{1}^{\prime \prime}=2 n_{1}^{\prime} n_{1}^{\prime \prime}$. Equation (16) can be obtained from dispersion equation (11) at $h=0$.

At a decrease in thickness of the metal film, the difference between the Bessel surface plasmon polariton mode effective indices increases. So, for example, in the structure containing gold $(\mathrm{Au})$ metal film of $20 \mathrm{~nm}$ thickness embedded in SF10 glass, the effective indices take on the values $n_{1}^{*}=1.759+$ $0.0018 i$ and $n_{2}^{*}=3.212+0.234 i$. At a metal film thickness of $15 \mathrm{~nm}$, we have $n_{1}^{*}=1.744+0.0009 i$ and $n_{2}^{*}=3.940+0.331 i$. As is seen, in the first case the generated high-index Bessel surface plasmon polariton has the first ring radius $R_{1}$ equal to $75 \mathrm{~nm}$. In the second case, while decreasing the thickness of metal film by $15 \mathrm{~nm}$, it is possible to obtain HIBSPP with $R_{1} \approx 61 \mathrm{~nm}$.

Taking into account that $\varepsilon_{1}^{\prime \prime}$ is significantly less than $\left|\varepsilon_{1}^{\prime}\right|$, (16) enables one to get the expressions for the real $\left(n_{\text {free }}^{* \prime}\right)$ and

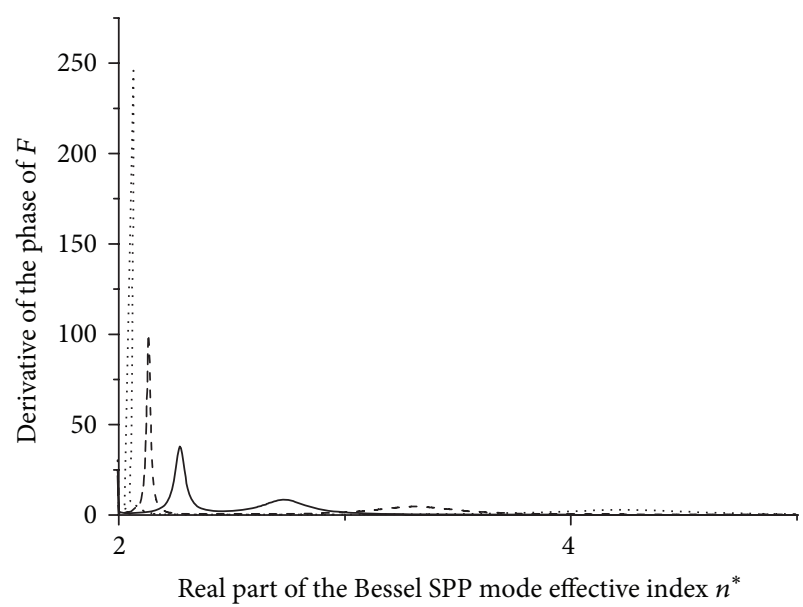

FIgure 6: The derivative of the phase of $F$ function versus the real part of Bessel surface plasmon polariton mode effective index for the gold film of $50 \mathrm{~nm}(-), 30 \mathrm{~nm}(--)$, and $20 \mathrm{~nm}(\cdots)$ embedded in $\mathrm{ZrO}_{2}$.

imaginary $\left(n_{\text {free }}^{* \prime}\right)$ parts of the effective index $n_{\text {free }}^{*}$ for Bessel surface plasmon polariton mode exited at the boundary of two semi-infinite media-dielectric and metal:

$$
n_{\text {free }}^{* \prime}=\sqrt{\frac{\varepsilon_{1}^{\prime} \varepsilon_{2}}{\varepsilon_{1}^{\prime}+\varepsilon_{2}}}, \quad n_{\text {free }}^{* \prime \prime}=\sqrt{\left(\frac{\varepsilon_{1}^{\prime} \varepsilon_{2}}{\varepsilon_{1}^{\prime}+\varepsilon_{2}}\right)^{3}} \frac{\varepsilon_{1}^{\prime \prime}}{2 \varepsilon_{1}^{\prime 2}} .
$$

The calculation, made according to (17), shows that for Bessel surface plasmon polariton, formed at the boundary of two semi-infinite media "gold-SF10 glass", $n_{\text {free }}^{* \prime \prime}=0.034$. As established above, for the structure containing the $50 \mathrm{~nm}$ thickness gold film embedded in SF10 glass, $\operatorname{Im}\left(n_{1}^{*}\right)=0.012$. Thus, in a thin metal film immerged in a dielectric, there may be the generation of a low-index Bessel surface plasmon polariton, for which the imaging part of the Bessel surface plasmon polariton mode effective index appears to be lower than that for BSPP excited at the boundary of semi-infinite media "metal-dielectric."

As it is mentioned above, in the $z$-direction, the electric field of the Bessel surface plasmon polariton decays as $\propto$ $\exp \left(-\operatorname{Im} k_{z i} z\right)(i=0,1,2)$. The penetration depth of the field in the medium is determined by the expression $d=1 / \operatorname{Im} k_{z i}$. As the calculation shows, for the LIBSPP penetration depth $d_{\text {LIBSPP }}$ into the targeted medium turns out to be larger than $d_{\text {free }}$ for the case of the plasmon formation at the "metaldielectric" boundary. So, in the case under study $d_{\text {LIBSPP }}=$ $137 \mathrm{~nm}, d_{\text {free }}=75 \mathrm{~nm}$.

Now using the reflection pole method, we consider the Bessel surface plasmon polaritons generation for a gold film of $50 \mathrm{~nm}$ thickness embedded in material for which the difference $\left|\varepsilon_{1}\right|-\varepsilon_{0}$ is lower than that in the case studied earlier. Such parameters belong, for example, to $\mathrm{ZrO}_{2}\left(\varepsilon_{0}=\right.$ $\left.\varepsilon_{2}=4\right)$. Figure 6 is the derivative of the phase of $F$ function versus the real part of the Bessel surface plasmon polariton mode effective index for this case. We see that there are two fast changes of the phase of $F$ corresponding to two Bessel surface plasmon polariton modes with the effective indices 


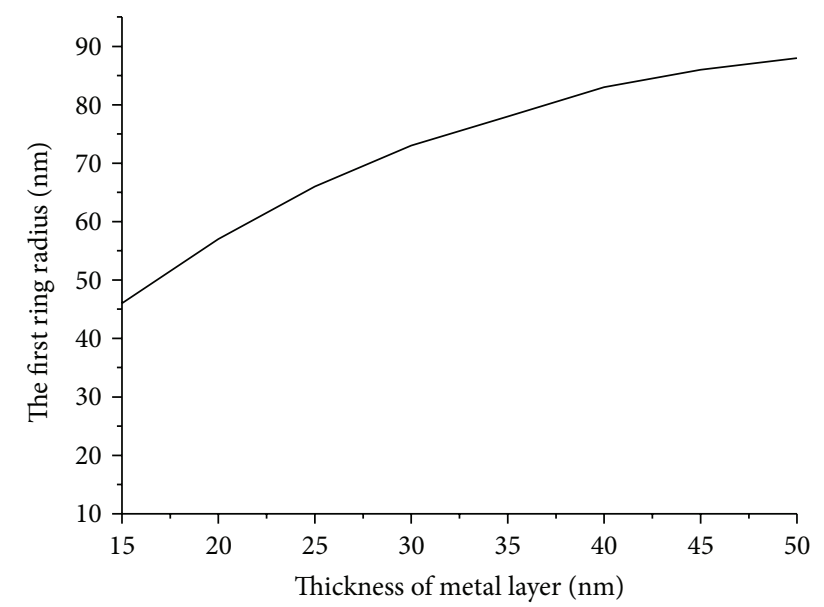

FIgURE 7: Dependence of the first ring radius of Bessel surface plasmon polariton on the thickness of Au layer embedded in $\mathrm{ZrO}_{2}$ for high-index BSPP.

dependent on the thickness $h$ of the metal layer. As it follows from Figure 7, for $h=50 \mathrm{~nm}$, we have $n_{1}^{*}=2.270+0.027 i$ and $n_{2}^{*}=2.732+0.120 i$; for $h=30 \mathrm{~nm}$, we obtain $n_{1}^{*}=$ $2.1320+0.0097 i$ and $n_{2}^{*}=3.312+0.217 i$; for $h=20 \mathrm{~nm}$, we find $n_{1}^{*}=2.065+0.004 i$ and $n_{2}^{*}=4.235+0.351 i$. It is seen that if the difference between the dielectric constants of a targeted medium (substrate) and metal decreases, the Bessel surface plasmon polariton mode effective indices increase. Moreover, if $h$ decreases, the difference $\Delta n^{*}=n_{2}^{*}-n_{1}^{*}$ increases. Thus, in a thin metal layer with a symmetric dielectric surrounding, the generation of a Bessel surface plasmon polariton with the first ring radius of several tens of nanometers is possible (see Figure 7).

2.3. Formation of Bessel Surface Plasmon Polaritons in Metal Film with Asymmetric Dielectric Surrounding. The asymmetric configuration leads to more interesting phenomena. Using the reflection pole method for solving dispersion equation (11), we searched the Bessel surface plasmon polaritons for a gold metal film of $50 \mathrm{~nm}$ thickness separating SF10 glass and air. The complex Bessel surface plasmon polariton mode effective indices are found to be $n_{1}^{*}=1.0480+0.0001 i$ and $n_{2}^{*}=2.022+0.043 i$. Here, the profile of $E_{1}^{l}(z)$ changes essentially inside the metal film for both generated types of $\mathrm{TM}_{0}$ Bessel surface plasmon polaritons (Figure 8), that is, it losses symmetry with respect to the middle plane of the metal layer. Moreover, Figure 8 shows that there is a certain value $z_{e}$ on the BSPP axis $(\rho=0)$, for which the longitudinal component of electrical vector $\vec{E}_{1}^{l}$ of high-index BSPP is equal to zero, in this concrete case $z_{e}=45 \mathrm{~nm}$.

As a result of the calculation performed with the help of the reflection pole method for solving dispersion equation (11), it is revealed that at an increase in the refraction index of the targeted medium Bessel surface plasmon polariton mode effective indices increase too, but their difference is determined, mainly, by the thickness of the metal film. And the value $z_{e}$ decreases.

\section{Intensity Distribution of Superposition of Bessel Surface Plasmon Polaritons in Layered Metal-Dielectric Structure}

Let us consider now the intensity distribution of the superposition of two $\mathrm{TM}_{m}$ Bessel surface plasmon polaritons with different topological charges $\left(m_{1} \neq m_{2}\right)$ and phase changes $\left(\Delta_{1} \neq \Delta_{2}\right)$ inside the targeted medium:

$$
\begin{aligned}
& I \sim\left|\vec{E}^{l}(R)\right|^{2}, \\
& \begin{aligned}
\vec{E}^{l}(R)= & \frac{A_{\mathrm{inc}}}{k_{0} n_{2}} \\
& \times\left[q_{1} t_{1} \exp i\right. \\
& \times\left[m_{1} \varphi+k_{z 2}^{(1)}\left(z-h_{1}\right)+\Delta_{1}\right] J_{m_{1}}\left(q_{1} \rho\right) \\
& +q_{2} t_{2} \exp i\left[m_{2} \varphi+k_{z 2}^{(2)}\left(z-h_{1}\right)+\Delta_{2}\right] \\
& \left.\times J_{m_{2}}\left(q_{2} \rho\right)\right] \vec{e}_{z} .
\end{aligned}
\end{aligned}
$$

Here, parameters $t_{1}, t_{2}$ determine the amplitudes of interfering plasmons and can be calculated from (12), $k_{z 2}^{(1,2)}=$ $\sqrt{k_{0}^{2} n_{2}^{2}-q_{1,2}^{2}}$. We will consider in more detail the case of practical interest where Bessel surface plasmon polaritons are generated in a Au film separating SF10 glass and air. This case is described in Section 2.3. Two different variants are realizable: case 1 where BSPPs with different $n^{*}$ (and hence, q) interfere; case 2 when BSPPs with equal $n^{*}$ (and hence, q) but different topological charges and/or phase changes interfere. Now consider one more important case where interfering Bessel surface plasmon polaritons with the same mode effective indices have topological charges equal in value but different in sign. In this case, the superposition of two BSPPs $J_{m}$ and $J_{-m}$, leads to the excitation of Bessel surface multiplasmon polariton for which, as it follows from (18), the azimuthal modulation of intensity is brought about. Owing to this, the interference structure being a set of annular maxima is separated into substructures at a nanoscale transverse size. Figure 9 illustrates the 2D distribution of normalized intensity in the air at the distance $z=\lambda / 3 \mu \mathrm{m}$ from the boundary with metal layer. Here, the plasmon polariton field is a superposition of $J_{3}$ and $J_{-3}$ low-index (a) and high-index (b) BSPPs. Figures 9 (c) and 9 (d) show that the field formed by two interfering high-index Bessel surface plasmon polaritons is characterized by more narrow near-axial maxima and essential suppression of lateral maxima while moving off the field axis to its periphery. The above-mentioned interference patterns are rather stable when moving off the interface of metal film. In Figure 10, as an example, the 2D normalized intensity distribution of the field formed by $J_{3}$ and $J_{-3}$ lowindex BSPPs is given at various distances from the "metal-air" boundary.

Thus, the superposition of two oppositely charged Bessel surface plasmon polaritons allows one to form near the interface of metal an array of quasi-nondiffracting nanoscale spots. 


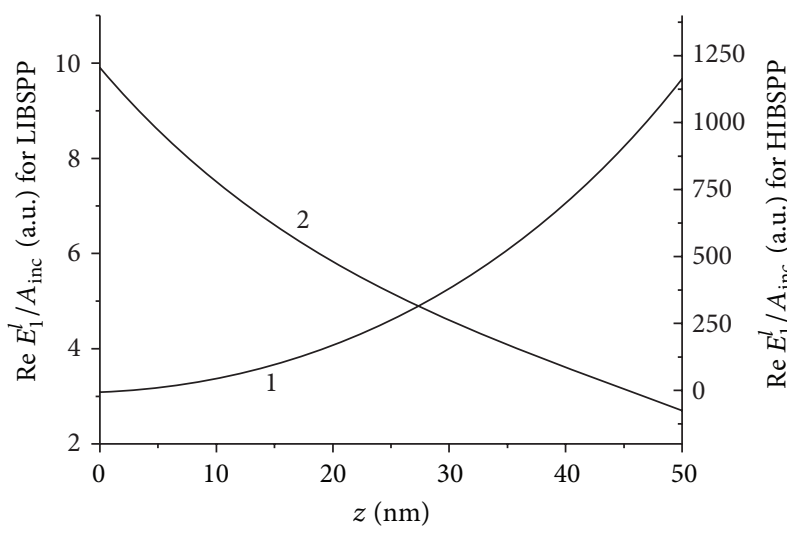

(a)

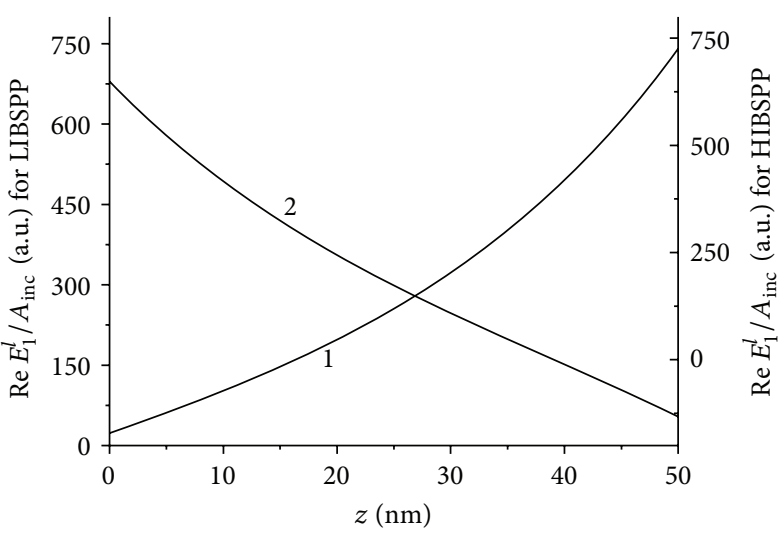

(b)

Figure 8: The normalized real part of the longitudinal component $E_{1}^{l}$ of electric vector of low-index (1) and high-index (2) TM0 Bessel surface plasmon polariton versus the distance inside the $50 \mathrm{~nm}$ gold film separating the SF10 glass and air (a) or fluid with refractive index 1.33 (b). The distance from the Bessel surface plasmon polariton axis is $\rho=0$, and the wavelength is $\lambda=0.633 \mu \mathrm{m}$.

\section{Possible Applications of a Superposition of Bessel Surface Plasmon Polaritons}

Thus, the established property of a superposition of Bessel surface plasmon polaritons is applicable to the creation of a virtual multitip for near-field optics. A conceptual sketch of formation of such a multitip, the so-called virtual Bessel surface multiplasmon polariton tip, is illustrated in Figures 11 and 12 .

As is seen from Figure 11, the lateral surface of conical lens 2 is illuminated by two conical beams (their extreme rays 1 and $1^{\prime}$ are presented in Figure 11) having the same halfcone angles. These beams are necessary for further exiting BSPPs. One of the pointed conical beams is obtained from the $\mathrm{TM}_{m}$ Bessel mode, and another conical beam is formed from the $\mathrm{TM}_{-m}$ BLB using a scheme represented in Figure 12 [39] and composed of a reflective conical lens (RCL) and conical mirror (CM). Both conical beams incident on the conical lens 2 perturb Bessel surface plasmon polaritons in metallic film 3. In the region of overlapping of conical beams (shaded area in Figure 11), a superposition of two Bessel surface plasmon polaritons is generated. In turn, in the air near the base surface of the conical lens covered by metal layer 3 , a Bessel surface multiplasmon polariton is formed. If the tested surface is placed at the distance $z \leq \lambda$ from the metal layer, the forming exponentially decaying field penetrates into the surface. Thus, the superposition of Bessel surface plasmon polaritons can be used for testing the surfaces of various specimens.

The first crucial advantage of the near-field technique based on a superposition of Bessel surface plasmon polaritons is related to the nondiffractional nature of these fields. Namely, the virtual Bessel surface multiplasmon polariton tip is characterized by the constant size of a probing beam while increasing the probe-to-sample distance which can vary from zero up to $\sim \lambda$. Thus, the use of the virtual Bessel surface multiplasmon polariton tip allows one to extend potentially the working distance between an object and apparatus.

The important feasible application of beams under consideration is also creating multiple optical traps when using two interfering high-order Bessel surface plasmon polaritons.

\section{Conclusion}

In this paper, a theory is developed of the generation of single Bessel SPPs and their superposition in the structure including a metal layer separating two semi-infinite dielectrics.

The comparison was made of Bessel surface plasmon polaritons investigated in this paper and traditional surface plasmon polaritons. The traditional SPP is a propagating wave process on the metal/dielectric surface. Unlike it, Bessel SPP is a superposition of counter propagating SPPs in all the possible radial directions. It is a complex highsymmetric interference light structure in sections parallel to the metal/dielectric interface. It should be noted as opposed to the propagating surface plasmon polariton, the Bessel one is a standing light structure.

The problem is studied of attenuation of the Bessel SPP excited by a limited narrow Bessel beam. It is shown that outside the region of the exciting source the Bessel SPP decays exponentially in the radial direction. It is established that the $1 / e$ energy-attenuation radius of Bessel SPP, generated by the limited narrow Bessel beam, coincides with the decay length of the propagating surface plasmon.

We have analyzed the cases of symmetric and asymmetric "dielectric-metal-dielectric" structures. A dispersion equation has been derived and numerically analyzed for these cases. The possibility is shown of excitation of two types (low- and high-index) of Bessel surface plasmon polaritons characterized by various profiles of longitudinal component $E^{l}(z)$ of electric vector inside the metal film. It has been 


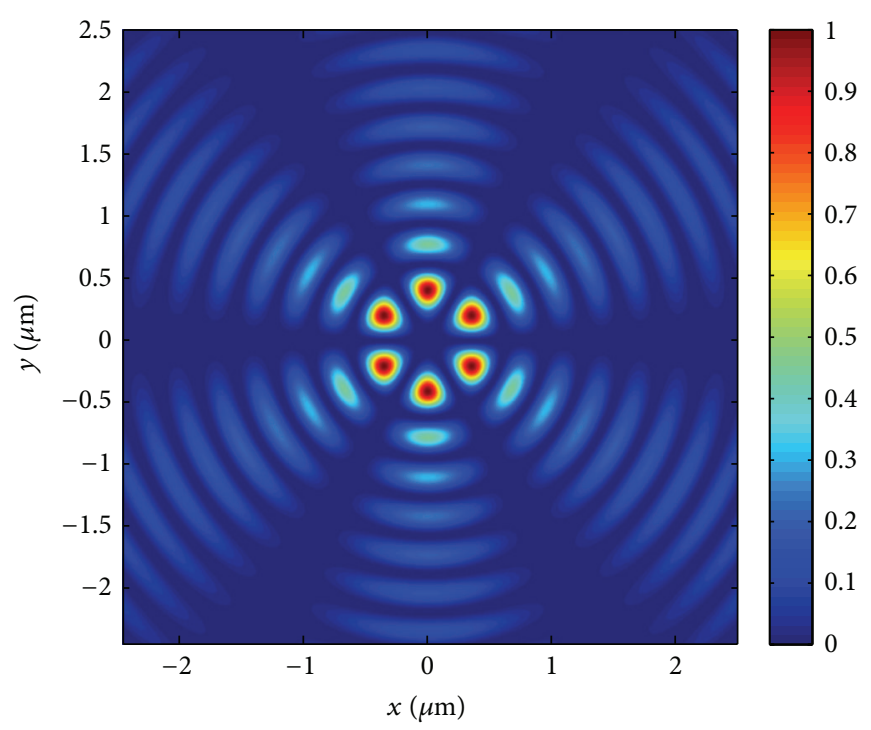

(a)

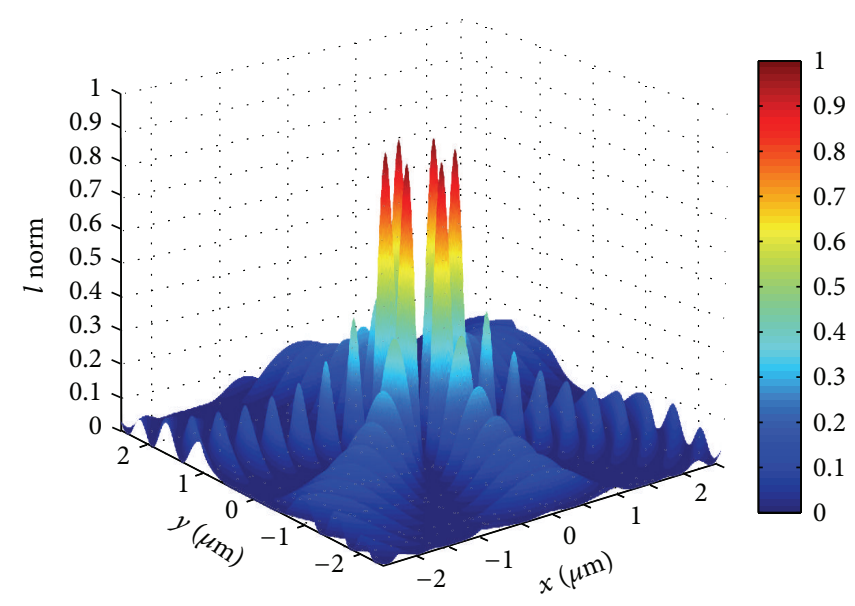

(c)

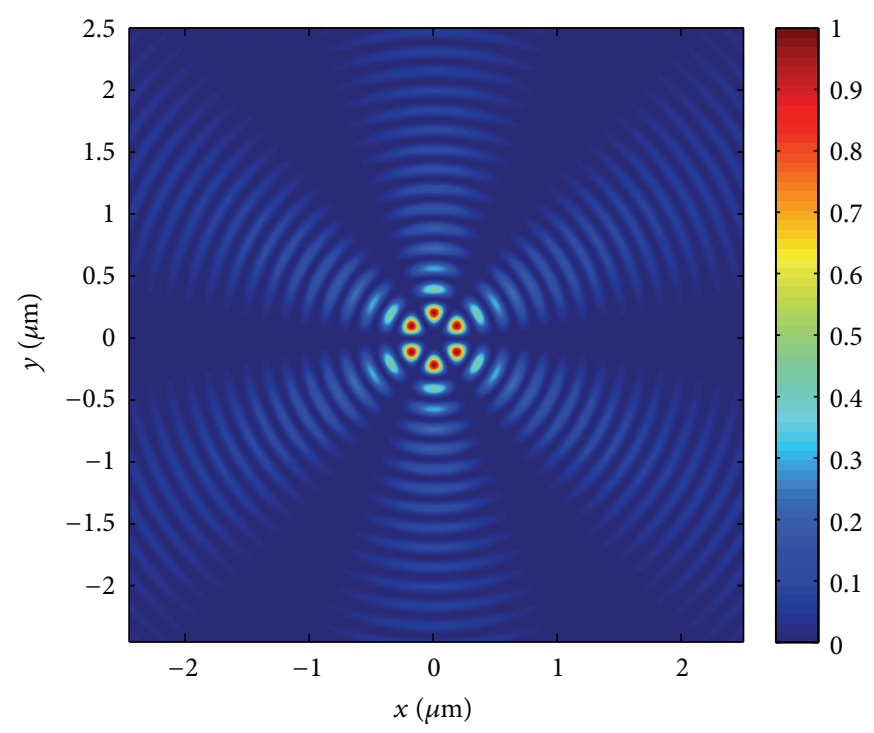

(b)

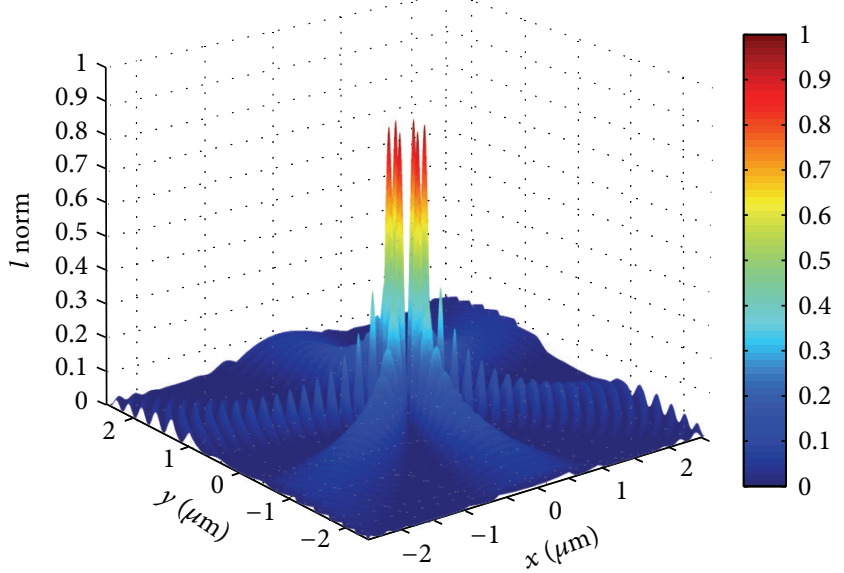

(d)

FIGURE 9: 2D and 3D normalized intensity distribution $\left|E_{2}^{l}\right|^{2} /\left|E_{2}^{l}\right|_{\max }^{2}$ of Bessel multiplasmon in the air at the distance $z=\lambda / 3 \mu \mathrm{m}$ from the interface of $50 \mathrm{~nm}$ thickness gold film of "SF10 glass-Au layer-air" structure. Bessel surface multiplasmon polariton is a superposition of $J_{3}$ and $J_{-3}$ low-index (a, c) and high-index (b, d) BSPPs with wavelength $\lambda=0.633 \mu \mathrm{m}$.

established that in a symmetric "dielectric-metal-dielectric" structure, the profile of $E^{l}(z)$ for low-index Bessel surface plasmon polaritons turns out to be a symmetric relatively medium plane of the metal film. For high-index Bessel surface plasmon polariton mode it is antisymmetric. In an asymmetric "dielectric-metal-dielectric" structure, the distribution of $E^{l}(z)$ inside metal film losses symmetry with respect to the medium plane of the metal layer for both generated plasmon polaritons. Moreover, there is a definite value $z_{e}$ of the longitudinal coordinate inside the layer, for which the longitudinal component of the electric vector $\vec{E}^{l}$ for high-index Bessel SPP is equal to zero. It is shown that if the difference between the refraction indices of adjoining dielectrics and a metal layer decreases, the position $z_{e}$ shifts toward the medium plane of metal film.

The dependence has been investigated of conditions of excitation of Bessel surface plasmon polaritons on the metal layer thickness $h$. It has been shown that if $h$ increases, the degeneration of Bessel plasmon modes occur. Here, the Bessel surface plasmon polariton mode effective index approaches the one for the case of excitation of Bessel SPP at the boundary of two semi-infinite media, that is, dielectric and metal.

The intensity distribution of low- and high-index Bessel surface plasmon polaritons inside and outside the metal layer have been calculated and analyzed. A two- and threedimensional pattern of the intensity for the single Bessel SPPs as well as for a superposition of two Bessel SPPs with opposite 


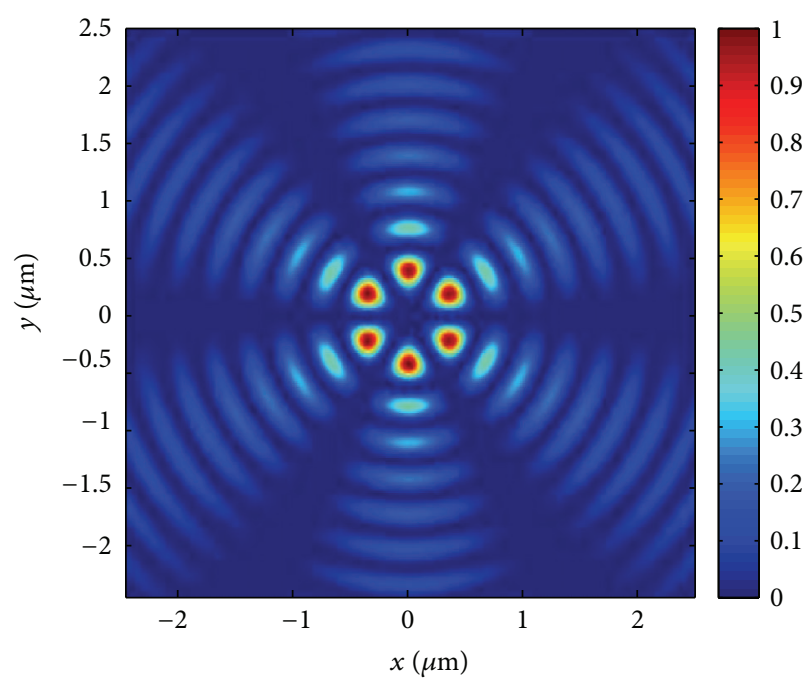

(a)

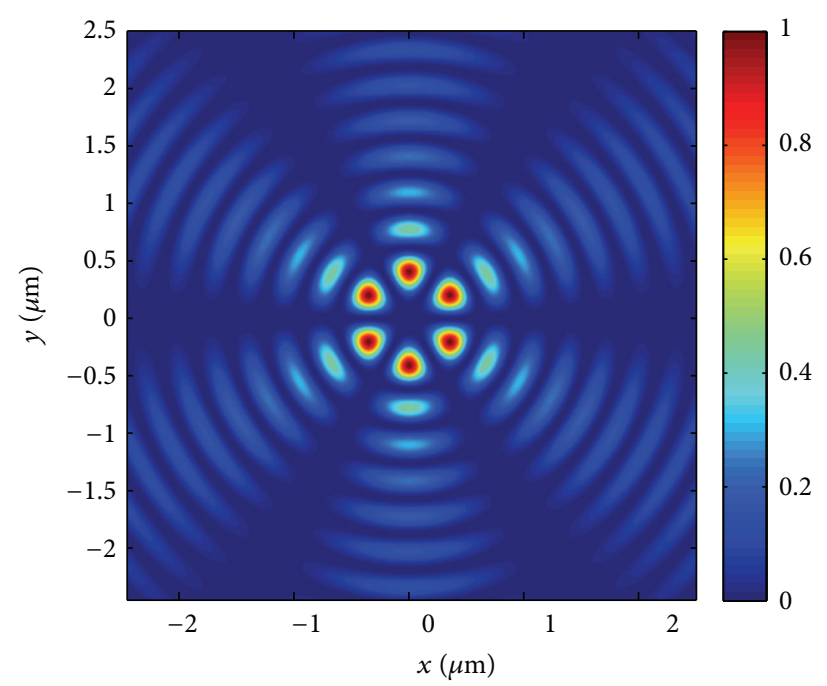

(b)

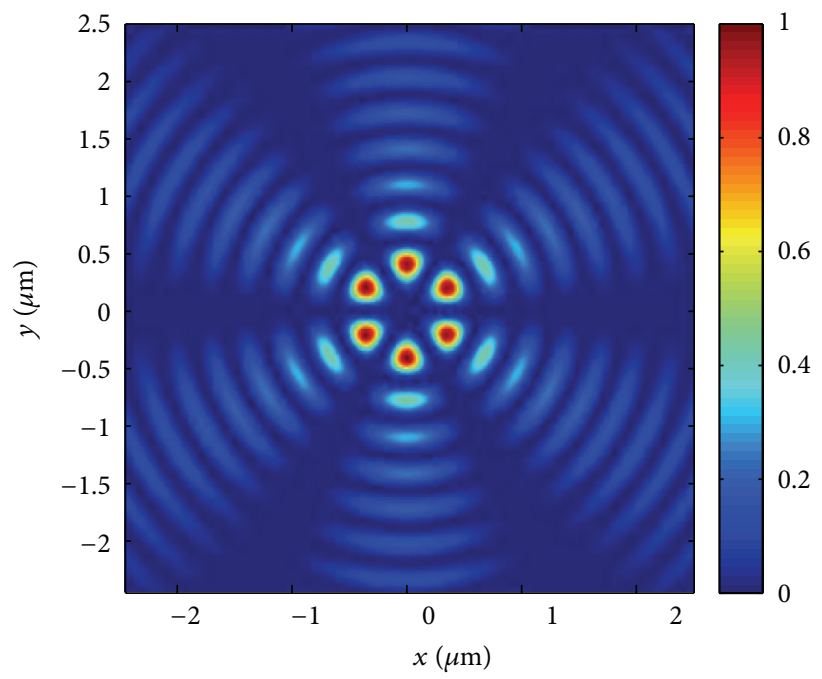

(c)

FIGURE 10: 2D normalized intensity distribution $\left|E_{2}^{l}\right|^{2} /\left|E_{2}^{l}\right|_{\max }^{2}$ of Bessel surface multiplasmon polariton in the air at the distance $z=\lambda / 3 \mu \mathrm{m}$ (a), $z=\lambda / 2 \mu \mathrm{m}$ (b), and $z=\lambda \mu \mathrm{m}$ (c) from the interface of $50 \mathrm{~nm}$ thickness gold film of "SF10 glass-Au layer-air" structure. Bessel surface multiplasmon polariton is a superposition of $J_{3}$ and $J_{-3}$ low-index BSPPs with wavelength $\lambda=0.633 \mu \mathrm{m}$.

topological charges has been calculated. It is shown that while increasing the distance from the surface of the metal layer, the central peak of Bessel SPP generated in the structure decays exponentially, but its transversal size does not change.

It is established that the plasmon polariton field, excited by the superposition of two Bessel beams with opposite topological charges, is characterized by the azimuthal modulation of intensity. Owing to it, the intensity pattern is separated into substructures of a nanoscale transverse size. It is shown that the above-mentioned substructures are rather stable when moving off the interface. Thus, using the superposition of two opposite charged vortex Bessel SPPs, which keeps their transversal size at any distance ranging from zero up to about the wavelength, one can form near the interface an array of diffraction-free nanoscale light "needles." This field is associated with creating a Bessel surface plasmon polariton multitip which is perspective for probing the surface simultaneously through several channels. An experimental set-up is proposed for producing such a Bessel surface plasmon polariton multitip.

The results obtained can be used for the development and optimization of techniques and devices for testing the quality of the surface of various substrates by Bessel surface plasmon polaritons; for creating Bessel surface plasmon polaritonbased optical tweezing systems; and for the capturing and manipulating of nanoparticles; applications are expected to be found in near-field optical microscopy with a nanoscale resolution. 


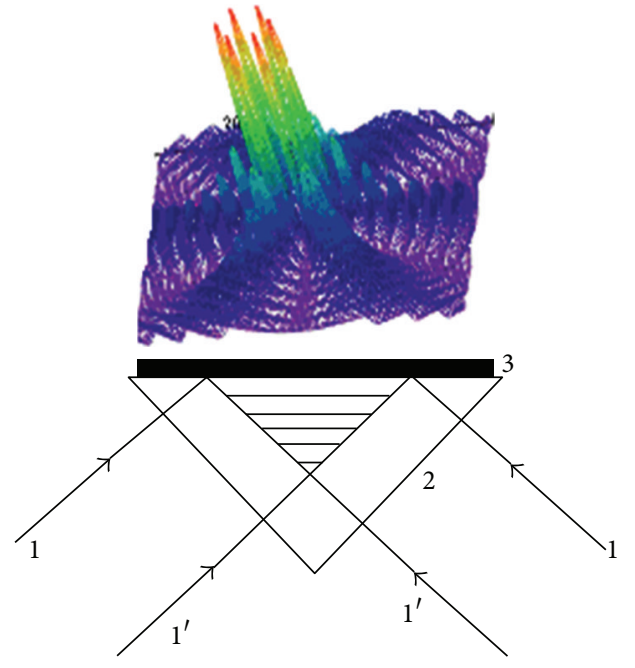

FIGURE 11: Schematic set-up for excitation of Bessel surface multiplasmon polariton. 1 and $1^{\prime}$ are extreme rays of conical beams excited Bessel surface plasmon polaritons, and 2 is conical lens on the basic surface of which metal film 3 is plotted.

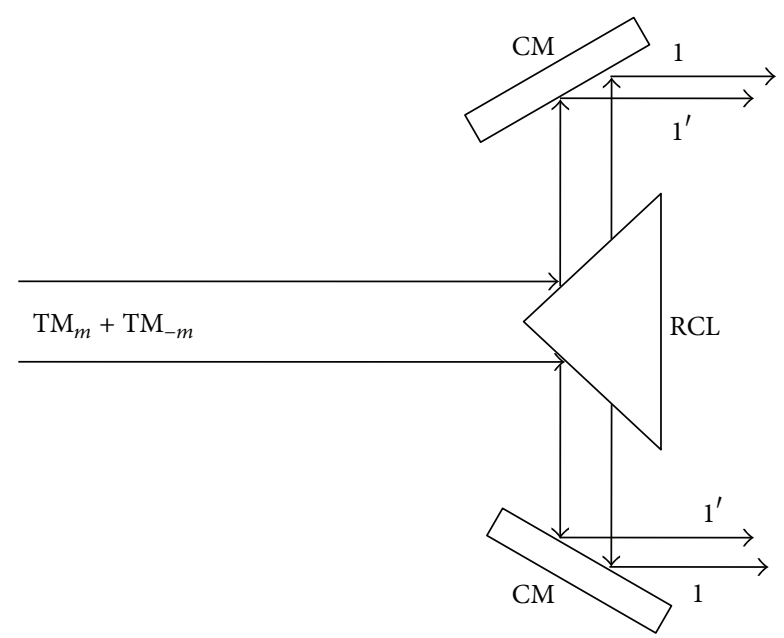

FIGURE 12: Basic scheme of obtaining of conical beams. RCL is a reflective conical lens, $\mathrm{CM}$ is a conical mirror, 1 and $1^{\prime}$ are extreme rays of the formed conical beam.

\section{References}

[1] H. Raether, Surface Plasmons on Smooth and Rough Surfaces and on Gratings, Springer, Berlin, Germany, 1988.

[2] E. N. Economou, "Surface plasmons in thin films," Physical Review, vol. 182, pp. 539-554, 1969.

[3] J. J. Burke, G. I. Stegeman, and T. Tamir, "Surface-polariton-like waves guided by thin, lossy metal films," Physical Review B, vol. 33, no. 8, pp. 5186-5201, 1986.

[4] W. L. Barnes, A. Dereux, and T. W. Ebbesen, "Surface plasmon subwavelength optics," Nature, vol. 424, no. 6950, pp. 824-830, 2003.

[5] A. V. Zayats, I. I. Smolyaninov, and A. A. Maradudin, "Nanooptics of surface plas-mon polaritons," Physics Reports, vol. 408, pp. 131-134, 2005.
[6] J. A. Sánchez-Gil and A. A. Maradudin, "Near-field and far-field scattering of surface plasmon polaritons by one-dimensional surface defects," Physical Review B, vol. 60, no. 11, pp. 8359-8367, 1999.

[7] J. Durnin, "Exact solutions for nondiffracting beams. I. The scalar theory," Journal of the Optical Society of America A, vol. 4, pp. 651-654, 1987.

[8] J. Durnin, J. J. Muceli, and J. H. Eberly, "Diffraction-free beams," Physical Review Letters, vol. 58, pp. 1499-1501, 1987.

[9] P. Sprangle and B. Hafizi, "Comment on nondiffracting beams," Physical Review Letters, vol. 66, pp. 837-839, 1991.

[10] Z. Bouchal, J. Wagner, and M. Chlup, "Self-reconstruction of a distorted non-diffracting beam," Optics Communications, vol. 151, pp. 207-211, 1998.

[11] Y. Lin, W. Seka, J. H. Eberly et al., "Experimental investigation of Bessel beam characteristics," Applied Optics, vol. 31, pp. 27082713, 1992.

[12] D. McGloin and K. Dholakia, "Bessel beams: diffraction in a new light," Contemporary Physics, vol. 46, pp. 15-28, 2005.

[13] J. Turunen and A. T. Friberg, "Self-imaging and propagationinvariance in electromagnetic fields," Pure and Applied Optics, vol. 2, pp. 51-60, 1993.

[14] R. Horak, Z. Bouchal, and J. Bajer, "Nondiffracting stationary electromagnetic field," Optics Communications, vol. 133, pp. 315327, 1997.

[15] T. A. Fadeyeva, C. N. Alexeyev, P. M. Anischenko, and A. V. Volyar, "Engineering of the space-variant linear polarization of vortex-beams in biaxially induced crystals," Applied Optics, vol. 51, pp. C224-C230, 2012.

[16] I. A. Litvin and A. Forbes, "Bessel Gauss resonator with internal amplitude filter," Optics Communications, vol. 281, pp. 23852390, 2008.

[17] V. N. Belyi, N. S. Kazak, S. N. Kurilkina, and N. A. Khilo, "Generation of TE- and TH-polarized Bessel beams using onedimensional photonic crystal," Optics Communications, vol. 282, no. 10, pp. 1998-2008, 2009.

[18] S. N. Kurilkina, V. N. Belyi, and N. S. Kazak, "Transformation of high-order Bessel vortices in one-dimensional photonic crystals," Journal of Optics A, vol. 12, no. 1, Article ID 015704, 12 pages, 2010.

[19] V. Garcés-Chávez, D. McGloin, H. Melville et al., "Simultaneous micromanipulation in multiple planes using a selfreconstructing light beam," Nature, vol. 419, pp. 145-147, 2002.

[20] J. Jerel, T. Cizmar, V. Nedela, and P. Zemanek, "Formation of long and thin polymer fiber using nondiffracting beam," Optics Express, vol. 14, pp. 8506-8515, 2006.

[21] M. K. Bhuyan, F. Courvoisier, P. A. Lacourt et al., "High aspect ratio taper-free microchannel fabrication using femtosecond Bessel beams," Optics Express, vol. 18, no. 2, pp. 566-574, 2010.

[22] B. Hafizi, E. Esarey, and P. Sprangle, "Laser-driven acceleration with Bessel beams," Physical Review E, vol. 55, no. 3, pp. 35393545, 1997.

[23] S. Rushin and A. Leizer, "Evanescent Bessel beams," Journal of the Optical Society of America A, vol. 15, pp. 1139-1143, 1998.

[24] S. N. Kurilkina, V. N. Belyi, and N. S. Kazak, "Features of evanescent Bessel light beams formed in structures containing a dielectric layer," Optics Communications, vol. 283, no. 20, pp. 3860-3868, 2010.

[25] A. M. Goncharenko, N. A. Khilo, and E. S. Petrova, "Evanescent Bessel light beams," in Lightmetry: Metrology, Spectroscopy, and 
Testing Techniques Using Light, vol. 4517 of Proceedings of SPIE, pp. 95-99, 2001.

[26] Q. Zhan, "Evanescent Bessel beam generation via surface plasmon resonance excitation by a radially polarized beam," Optics Letters, vol. 31, no. 11, pp. 1726-1728, 2006.

[27] J. Xi, Q. Li, and J. Wang, "Numerical simulation of evanescent Bessel beams and apodization of evanescent field in near-field optical virtual probe," in Nanophotonics, Nanostructure, and Nanometrology, Proceedings of SPIE, pp. 42-51, 2005.

[28] A. V. Novitsky and L. M. Barkovsky, "Total internal reflection of vector Bessel beams: imbert-Fedorov shift and intensity transformation," Journal of Optics A, vol. 10, Article ID 075006, 7 pages, 2008.

[29] M. K. Al-Muhanna, S. N. Kurilkina, V. N. Belyi, and N. S. Kazak, "Energy flow patterns in an optical field formed by a superposition of evanescent Bessel light beams," Journal of Optics, vol. 13, no. 10, Article ID 105703, 2011.

[30] T. Grosjean, D. Courjon, and D. Van Labeke, "Bessel beams as virtual tips for near-field optics," Journal of Microscopy, vol. 210, no. 3, pp. 319-323, 2003.

[31] H. Kano, D. Nomura, and H. Shibuya, "Excitation of surfaceplasmon polaritons by use of a zeroth-order Bessel beam," Applied Optics, vol. 43, no. 12, pp. 2409-2411, 2004.

[32] K. J. Moh, X. C. Yuan, J. Bu, S. W. Zhu, and B. Z. Gao, "Radial polarization induced surface plasmon virtual probe for twophoton fluorescence microscopy," Optics Letters, vol. 34, no. 7, pp. 971-973, 2009.

[33] C. J. Zapata-Rodriguez, S. Vuković, M. R. Belić, D. Pastor, and J. J. Miret, "Nondiffracting Bessel plasmons," Optics Express, vol. 19, pp. 19572-19581, 2011.

[34] M. Born and E. Wolf, Principles of Optics, Cambridge University Press, Cambridge, UK, 1999.

[35] G. N. Watson, A Threatise on the Theory of Bessel Functions, Cambridge University Press, Cambridge, UK, 1966.

[36] G. B. Arfken, H. J. Weber, and F. E. Harris, Mathematical Methods for Physicists, Academic Press, Orlando, Fla, USA, 1985.

[37] A. Papoulis, Circuits and Systems, Holt, Rinehart and Winston, New York, NY, USA, 1980.

[38] M. Yamamoto, "Surface plasmon resonance. Theory: tutorial," Review of Polarography, vol. 48, pp. 209-237, 2002.

[39] V. N. Belyi, N. A. Khilo, N. S. Kazak, A. A. Ryzhevich, and A. Forbes, "Propagation of high-order circularly polarized Bessel beams and vortex generation in uniaxial crystals," Optical Engineering, vol. 50, Article ID 059001, 9 pages, 2011. 

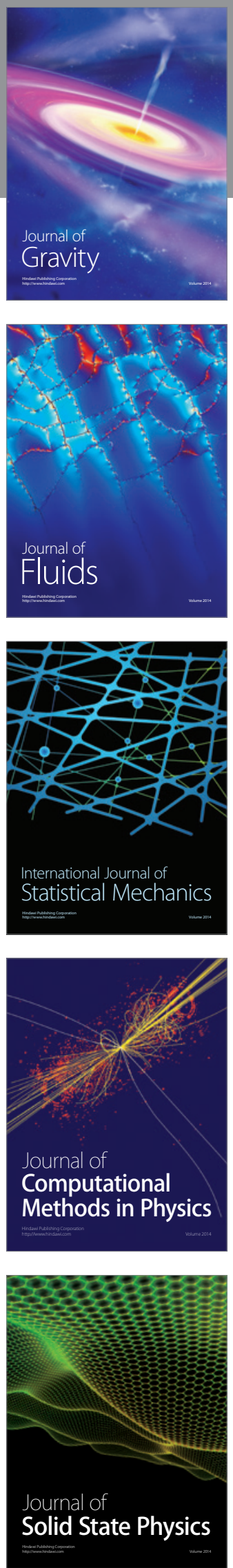

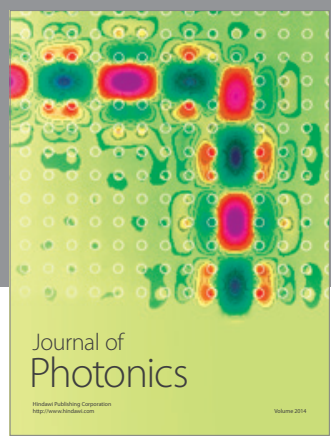

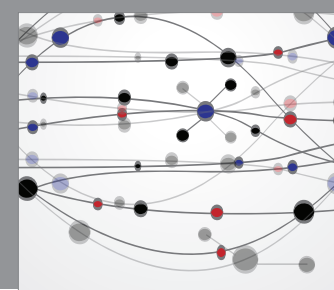

The Scientific World Journal

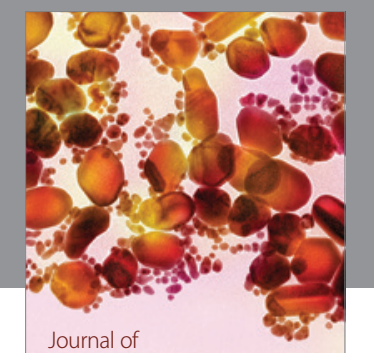

Soft Matter
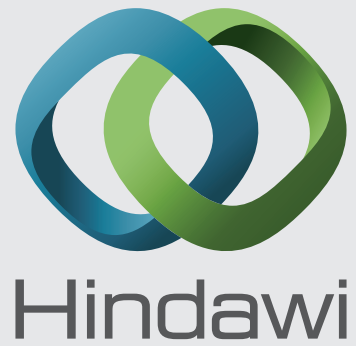

Submit your manuscripts at

http://www.hindawi.com
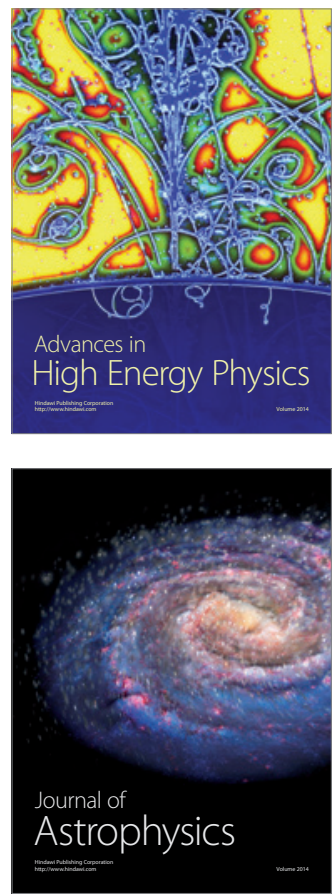
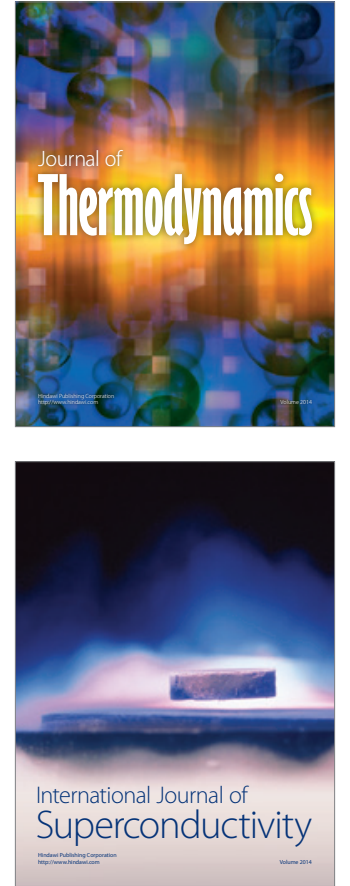
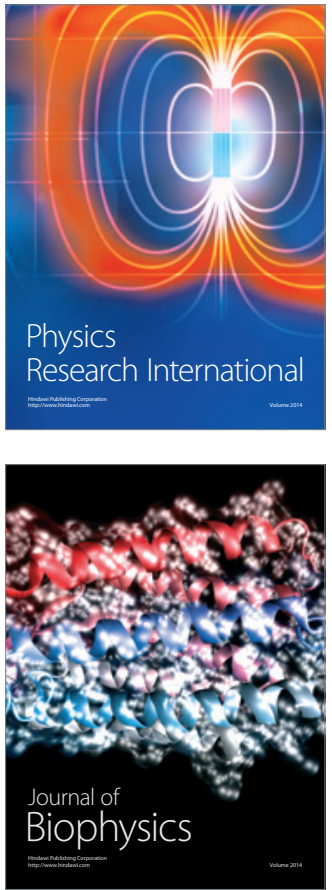
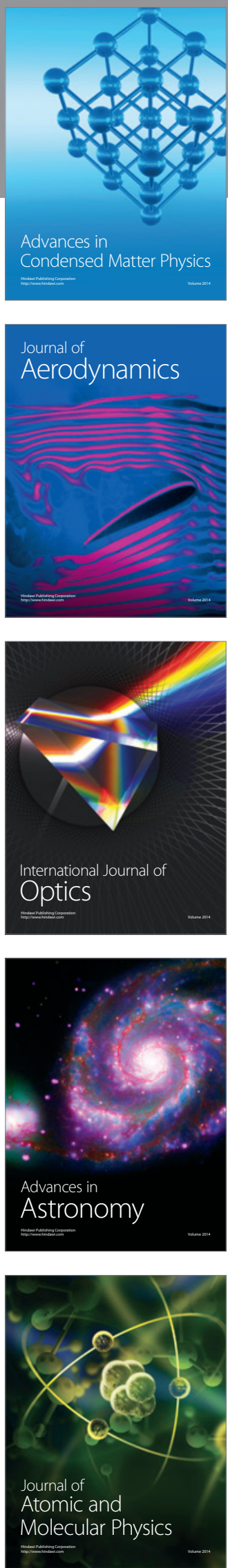